\title{
QUANTUM STOCHASTIC PROCESSES \\ ARISING FROM THE STRONG RESOLVENT LIMITS OF THE SCHRÖDINGER EVOLUTION IN FOCK SPACE
}

\author{
ALEXANDER M. CHEBOTAREV \\ Moscow State University, Faculty of Physics \\ Quantum Statistics Department, Moscow 119899, Russia \\ E-mail: alex@cheb.phys.msu.su \\ DMITRY V. VICTOROV \\ Moscow State University, Faculty of Physics \\ Quantum Statistics Department, Moscow 119899, Russia \\ E-mail:victorov@qs.phys.msu.su
}

\begin{abstract}
By using F. A. Berezin's canonical transformation method [5], we derive a nonadapted quantum stochastic differential equation (QSDE) as an equation for the strong limit of the family of unitary groups satisfying the Schrödinger equation with singularly degenerating Hamiltonians in Fock space. Stochastic differentials of QSDE generate a nonadapted associative Ito multiplication table, and the coefficients of these differentials satisfy the formal unitarity conditions of the Hudson-Parthasarathy type [10].
\end{abstract}

Introduction. The derivation of equations for quantum noise is an important problem related to applications in quantum optics, quantum information and measurement theories $[1,4,9,15]$. The main advantage of quantum stochastic theory is an elegant implicit construction of solutions for the class of adapted QSDE describing the joint unitary evolution of a quantum system and its environment [10]. The conceptual disadvantage of this approach is a gap in understanding the unitary stochastic evolution in the spirit of the classical Stone theorem describing the infinitesimal generator of a unitary group as a self-adjoint operator.

Until the recent papers $[7,8]$, it was not known that a unitary cocycle satisfying the QSDE can be described not by a symmetric operator but rather by a symmetrical bound-

1991 Mathematics Subject Classification: Primary 81S25; Secondary 47N50, 46L60.

Research supported by RFBR grant N 95-01-007784 and by grant of Goskomvus of Russian Federation N 96-1.7-100

The paper is in final form and no version of it will be published elsewhere. 
ary value problem. The boundary condition describes jumps of the solution in amplitude and in phase. The jumps are supported by the set of singularities of the formal generator, and the selfadjointness of the boundary value problem is necessary and sufficient for the existence of the unitary solution of the appropriate QSDE.

These results were obtained by the explicit analysis of the class of solvable problems for the Schrödinger equation in Fock space $[7,8]$. In the present paper, we extend the class of Hamiltonian operators by using the standard analytical tool: the Berezin canonical transformation technique.

In $\$ 1$ we recall the construction of the normal form of the resolving operator for the Schrödinger equation in Fock space. In $\S 2$ we consider a solvable model and describe explicitly the limit unitary group and the corresponding unitary cocycle which satisfies the adapted QSDE. In $\S 3$ we analyze the range of the resolvent (the domain of the infinitesimal operator) and derive boundary discontinuity conditions and the dissipative operator, which turns out to be symmetric on the linear subspace of Fock vectors satisfying the imposed boundary condition. In $\S 4$, by the asymptotic analysis of the normal form of the resolving operator, we prove similar results for the class of Hamiltonians containing squares of creation and annihilation operators. We also describe a nontrivial connection between the coefficients of the Hamiltonian operator and the coefficients of QSDE.

1. Berezin's approach to the Schrödinger equation in Fock space. In the sixties, F. A. Berezin has developed the technique of canonical transformations of CCR-CAR representations [5] which can be used to construct the resolving operator of the Cauchy problem for the Schrödinger equation in Fock space with the quadratic Hamiltonian

$$
H_{2}\left(a^{*}, a\right)=\frac{1}{2}\left\{\left(a^{*}, \bar{H}_{a a} a^{*}\right)+\left(a, H_{a a} a\right)+2\left(a^{*}, H_{a^{*} a} a\right)\right\}+\left(a^{*}, \bar{H}_{a}\right)+\left(a, H_{a}\right)+H_{0} .
$$

He proved an explicit formula for the normal symbol $U_{t}\left(a^{*}, a\right)$ of the resolving operator

$$
U_{t}\left(a^{\dagger}, a\right)=\exp \left\{i t H_{2}\left(a^{\dagger}, a\right)\right\},
$$

where the pairs $\left(a^{\dagger}, a\right)$ are the creation and annihilation operators in the symmetric Fock space $\Gamma^{S}\left(L_{2}(\mathbb{R})\right)$, and the pairs $\left(a^{*}, a\right)$ are functions from $L_{2}(\mathbb{R})$.

The theory involves the real inner product in $L_{2}(\mathbb{R})$ and three involutions assuming the representation of operators by integral kernels. These involutions are the Hermite conjugation (star*), the complex conjugation $(\overline{\mathrm{bar}})$ and the transposition (prime') such that $B^{*}=\bar{B}^{\prime}$.

One of the main assertions of the book [5] (Chap. III, $\S 6$, Th.2) is a theorem describing the construction of the normal form $U_{t}\left(a^{*}, a\right)$ of the resolving operator $U_{t}\left(a^{\dagger}, a\right)$ for the quadratic Hamiltonian $H_{2}\left(a^{*}, a\right)$ :

$$
U_{t}\left(a^{*}, a\right)=C_{t} \exp \left\{\frac{1}{2} \Sigma_{t}\left(a^{*}, a\right)-\left(a^{*}, a\right)\right\}, \quad C_{t}=\left\{\operatorname{det}\left(\Phi_{t} P_{t}\right)\right\}^{-1 / 2} e^{i S_{t}},
$$

where

$$
\mathcal{M}_{t}=\left(\begin{array}{ll}
\Phi_{t} & \Psi_{t} \\
\bar{\Psi}_{t} & \bar{\Phi}_{t}
\end{array}\right)=\exp \left\{i t\left(\begin{array}{cc}
-H_{a^{*} a}-\bar{H}_{a a}, \\
H_{a a} & \bar{H}_{a^{*} a}
\end{array}\right)\right\}, \quad \mathcal{N}_{t}=\left(\begin{array}{cc}
\bar{\Psi}_{t} \Phi_{t}^{-1} & \left(\Phi_{t}^{\prime}\right)^{-1} \\
\Phi_{t}^{-1} & -\Phi_{t}^{-1} \Psi_{t}
\end{array}\right),
$$




$$
\begin{gathered}
\Sigma_{t}=\left(\begin{array}{c}
a \\
a^{*}
\end{array}\right)^{\prime}\left(\mathcal{N}_{t}\left(\begin{array}{c}
a \\
a^{*}
\end{array}\right)+\left(\begin{array}{c}
\bar{g}_{t}-\bar{\Psi}_{t} \Phi_{t}^{-1} g_{t} \\
-\Phi_{t}^{-1} g_{t}
\end{array}\right)\right), \quad\left(\begin{array}{c}
g_{t} \\
\bar{g}_{t}
\end{array}\right)=i \int_{0}^{t} d \tau \mathcal{M}_{\tau}\left(\begin{array}{c}
-\bar{H}_{a} \\
H_{a}
\end{array}\right), \\
S_{t}=\int_{0}^{t} d \tau\left\{\left(\left(g_{\tau}\left(\Phi_{\tau}^{\prime}\right)^{-1} H_{a a}-H_{a}\right), \Phi_{\tau}^{-1} g_{\tau}+H_{0}\right\}, \quad P_{t}=e^{i t H_{a^{*} a}} .\right.
\end{gathered}
$$

The normal symbol (1.2) is well defined provided $\Psi_{t} \in \mathcal{T}_{2}\left(\mathcal{L}_{2}(\mathbb{R})\right) \subset \mathcal{B}\left(\mathcal{L}_{2}(\mathbb{R})\right)$, that is, $\Psi_{t}$ is a Hilbert-Schmidt operator, and $g_{t}(\omega) \in \mathcal{L}_{2}(\mathbb{R})$ for each $t \in \mathbb{R}_{+}$.

The normal forms of the Hamiltonian $H\left(a^{*}, a\right)$ and its resolving operator $U_{t}\left(a^{*}, a\right)$ are related by the Schrödinger equation

$$
\left\{\frac{d}{d t}-i H\left(a^{*}, a+\delta_{a^{*}}\right)\right\} U_{t}\left(a^{*}, a\right)=0,
$$

where the Fréchet derivative $\delta_{a^{*}}$ acts the first, and the multiplication by the function $a^{*}(\omega) \in \mathcal{L}_{2}(\Omega)$ acts the second.

2. The limits of solvable models. Consider the family of unitary groups

$$
U_{t}^{(\alpha)}=\exp \left\{i t H^{(\alpha)}\right\}, \quad \alpha \in \mathbb{R}_{+}
$$

generated by the family of selfadjoint Hamiltonians with commuting coefficients

$$
H^{(\alpha)}=I \otimes E+H_{0} \otimes I+R^{*} \otimes A\left(f_{\alpha}\right)+R \otimes A^{\dagger}\left(f_{\alpha}\right)+K \otimes A^{\dagger}\left(g_{\alpha}\right) A\left(g_{\alpha}\right),
$$

where $H_{0}$ and $E$

$$
E=\int_{-\infty}^{\infty} d \omega \omega a^{\dagger}(\omega) a(\omega)
$$

are the energies of the system and the environment, $K$ is a self-adjoint operator, $R$ is a closed operator, and $\left\{g_{\alpha}\right\}$ and $\left\{f_{\alpha}\right\}$ are sequences of functions with positive Fourier transforms from $L_{2}(\mathbb{R}) \cap L_{1}(\mathbb{R})$ degenerating to the constant $1 / \sqrt{2 \pi}$ as $\alpha \rightarrow 0$. The main example of dependence on $\alpha$ is given by the scaling transformation $g_{\alpha}(\omega)=g(\alpha \omega), g(\omega)=$ $\exp \left\{-\omega^{2}\right\} / \sqrt{2 \pi}$ (see [6]).

In the particular case $H_{2}\left(a^{*}, a\right)=\left(a^{*}, C a\right)+\left(a^{*}, f\right)+(\bar{f}, a)$, we have $H_{a a}=0$ and

$$
H_{a^{*} a}=C \cong I \otimes \omega_{1} \delta\left(\omega_{1}-\omega_{2}\right)+K \otimes\left\{\overline{g_{\alpha}\left(\omega_{1}\right)} g_{\alpha}\left(\omega_{2}\right)\right\}, \quad H_{a}=f \cong R^{*} \otimes f_{\alpha}(\omega) .
$$

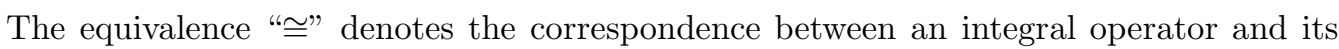
kernel, and between a vector from $\mathcal{H}$ and an appropriate function from $\mathcal{L}_{2}(\mathbb{R})=\mathcal{H}$.

In the sequel, we suppose for simplicity that the operators $H_{0}, K$ and $R$ are bounded and have a joint spectral family $E_{\lambda}$ such that

$$
H_{0}=\int \nu_{\lambda} d E_{\lambda}, \quad K=\int \lambda d E_{\lambda}, \quad R=\int \rho_{\lambda} e^{i \theta_{\lambda}} d E_{\lambda}, \quad \lambda \in \operatorname{Sp} K,
$$

where $\nu_{\lambda}, \rho_{\lambda}, \theta_{\lambda}$ are measurable real functions corresponding to the operators $H_{0}, K$ and $R$. These assumptions single out the class of simple solvable models with nontrivial behavior as $\alpha \rightarrow 0$.

Since $H_{a a}=0$, equations (1.3) imply:

$$
\Psi_{t}^{(\alpha)}=0, \quad\left(\Phi_{-t}^{(\alpha)}\right)^{\prime}=\Phi_{-t}^{(\alpha)}=P_{t}^{(\alpha)}=e^{i t H_{a^{*} a}^{(\alpha)}}=e^{i t\left(\omega+K \otimes\left|g_{\alpha}\right\rangle\left\langle g_{\alpha}\right|\right)},
$$




$$
C_{t}^{(\alpha)}=\operatorname{det}\left(\Phi_{t}^{(\alpha)} P_{t}^{(\alpha)}\right)^{-1 / 2}=1, \quad g_{t}^{(\alpha)}=-i R^{*} \int_{0}^{t} d \tau P_{-\tau}^{(\alpha)} f_{\alpha}
$$

and Eq. (1.2) reads as follows

$$
U_{t}^{(\alpha)}\left(a^{*}, a\right)=\exp \left\{\frac{1}{2} \Sigma_{t}^{(\alpha)}\left(a^{*}, a\right)+i S_{t}^{(\alpha)}-\left(a^{*}, a\right)\right\},
$$

where

$$
\begin{gathered}
S_{t}^{(\alpha)}=i \int_{0}^{t} d \tau \int_{0}^{\tau} d s R^{*}\left(\bar{f}_{\alpha}, P_{s}^{(\alpha)} f_{\alpha}\right) R+H_{0} t \\
\frac{1}{2} \Sigma_{t}^{(\alpha)}=\left(a^{*}, P_{t}^{(\alpha)} a\right)+i\left(a^{*}, \int_{0}^{t} d s P_{s}^{(\alpha)} f_{\alpha}\right) R+i R^{*}\left(\int_{0}^{t} d s \overline{P_{s}^{(\alpha)} f_{\alpha}}, a\right) .
\end{gathered}
$$

Equation (1.3) is satisfied for all $t>0$ and $\left.U_{t}\left(a^{*}, a\right)\right|_{t=0}=I$.

It is possible to describe explicitly the action of the resolving operator on direct products $h \otimes \psi(v)$, where $h \in \mathcal{H}, \psi(v) \in \Gamma^{S}\left(\mathcal{L}_{2}(\mathbb{R})\right), v \in \mathcal{L}_{2}(\mathbb{R})$, and $\psi(v)$ is a coherent (exponential) vector. The unitary group $U_{t}^{(\alpha)}=\exp \left\{i H^{(\alpha)} t\right\}$ with Hamiltonian (2.1) and normal form (2.2) acts on exponential vectors as follows:

$$
U_{t}^{(\alpha)} h \otimes \psi(v)=\int e^{i \nu_{\lambda} t} d E_{\lambda} h \otimes \psi\left(v_{t}^{(\alpha)}(\lambda)\right) \exp \left\{i \rho_{\lambda} e^{-i \theta_{\lambda}} \int_{0}^{t}\left(f_{\alpha}, v_{s}^{(\alpha)}(\lambda)\right) d s\right\},
$$

where $v_{t}^{(\alpha)}(\lambda)=P_{t}^{(\alpha)}(\lambda) v+i \rho_{\lambda} e^{i \theta_{\lambda}} \int_{0}^{t} P_{s}^{(\alpha)}(\lambda) f_{\alpha} d s$. We recall that we use the real inner product in $(2.3)$.

The limit of the derivative

$$
H_{w}=-i \lim _{\alpha \rightarrow 0}\left\{\left.\frac{d}{d t} e^{i t H^{(\alpha)}}\right|_{t=0}\right\}
$$

coincides clearly with the weak limit of Hamiltonians (1.1)

$$
H_{w}=I \otimes E+H_{0} \otimes I+R^{*} \otimes A(1)+R \otimes A^{\dagger}(1)+K \otimes A^{\dagger}(1) A(1)
$$

with the same operator coefficients $R$ and $K$. To find the derivative of the limit

$$
H_{\text {srs }}=-\left.i \frac{d}{d t}\left\{\lim _{\alpha \rightarrow 0} e^{i t H^{(\alpha)}}\right\}\right|_{t=0},
$$

which gives the limit of the generators in the strong resolvent sense, we use the expansion

$$
\exp \{i c \widehat{\pi}\}=I+\left(e^{i c}-1\right) \widehat{\pi}, \quad \widehat{\pi}=\widehat{\pi}^{*}=\widehat{\pi}^{2}, \quad c \in \mathbb{C}
$$

for any projector $\widehat{\pi}$, and the "Lemma on Four Limits" $[6,7]$ describing the singular limits of the components of functionals $\Sigma_{t}^{(\alpha)}$ and $S_{t}^{(\alpha)}$ in equation (2.3):

$$
\begin{gathered}
\lim \int_{0}^{t} d s\left(\bar{f}_{\alpha}, P_{s}^{(\alpha)} f_{\alpha}\right)=(2-i K)^{-1}, \\
\lim \left(a^{*}, \int_{0}^{t} d s P_{s}^{(\alpha)} f_{\alpha}\right)=\int_{0}^{t} \frac{d s}{\sqrt{2 \pi}} \int \frac{d \omega a^{*}(\omega) e^{i s \omega}}{I-i K / 2}=\frac{2}{(2-i K)}\left(a^{*}, e^{i \omega t} \mathcal{F}_{\tau \rightarrow \omega} I_{[0, t)}(\tau)\right), \\
\lim \left(\int_{0}^{t} d s \overline{P_{s}^{(\alpha)} f_{\alpha}}, a\right)=\int_{0}^{t} \frac{d s}{\sqrt{2 \pi}} \int \frac{d \omega a(\omega) e^{-i s \omega}}{I-i K / 2}=\frac{2}{(2-i K)}\left(\mathcal{F}_{\tau \rightarrow \omega} I_{[0, t)}(\tau), a\right),
\end{gathered}
$$




$$
s-\lim P_{t}^{(\alpha)}=e^{i t \omega}\left(I+(W-I) \widehat{\pi}_{[0, t)}\right) .
$$

By $\widehat{\pi}_{T}$ we denote the projection-valued set function $\widehat{\pi}_{T} v(\omega)=\mathcal{F}_{t \rightarrow \omega} I_{T}(t) \mathcal{F}_{\omega \rightarrow t}^{-1} v(\omega)$; $\mathcal{F}_{t \rightarrow \omega}$ is the Fourier transformation:

$$
\mathcal{F}_{t \rightarrow \omega} f=(2 \pi)^{-1 / 2} \int d t e^{-i \omega t} f(t),
$$

and $I_{T}(t)$ is the indicator function of the set $T$.

Substituting limits (2.6) into equation (2.2) for the normal form of the resolving operator, we obtain

with

$$
\lim _{\alpha \rightarrow 0} U_{t}^{(\alpha)}\left(a^{*}, a\right)=\exp \left\{Z_{t}\left(a^{*}, a\right)\right\}
$$

$$
\begin{gathered}
Z_{t}\left(a^{*}, a\right)=i t\left(H_{0}-R^{*}(2-i K)^{-1} R\right)+(W-I)\left(a^{*}, e^{i \omega t} \widehat{\pi}_{[0, t)} a\right)+\left(a^{*}, e^{i \omega t} a\right) \\
+i(I-i K / 2)^{-1} R\left(a^{*}, e^{i \omega t} \widetilde{I}_{[0, t)}\right)+i R^{*}(I-i K / 2)^{-1}\left(\widetilde{I}_{[0, t)}, a\right) .
\end{gathered}
$$

By differentiating (2.7), we find the normal form of the infinitesimal operator of the limit unitary group:

$$
i H_{\text {srs }}=i I \otimes E+L_{0} \otimes I+(W-I) \otimes A^{\dagger}(1) A(1)+L \otimes A^{\dagger}(1)-L^{*} W \otimes A(1)
$$

with $L=i(1-i K / 2)^{-1} R, L_{0}=i H_{0}-R^{*}(2-i K)^{-1} R$, and $W=(2+i K)(2-i K)^{-1}$. Thus, we conclude that there exist two different limits as $\alpha \rightarrow 0$ : the weak limit $H_{w}$ (2.4) and $H_{\text {srs }}$ which has the meaning of the strong resolvent limit of Hamiltonians (1.2):

$$
H_{\text {srs }}=I \otimes E+i^{-1}\left\{L_{0} \otimes I+L_{1} \otimes A(1)+L_{2} \otimes A^{\dagger}(1)+L_{3} \otimes A^{\dagger}(1) A(1)\right\}
$$

with the coefficients $L_{\ell}$,

$$
\begin{gathered}
L_{1}=R^{*} \frac{2 i}{2-i K}, \quad L_{2}=\frac{2 i}{2-i K} R, \quad L_{3}=\frac{2 i K}{2-i K}, \\
L_{0}=i H_{0}-R^{*} \frac{i K}{4+K^{2}} R-R^{*} \frac{2}{4+K^{2}} R
\end{gathered}
$$

satisfying exactly the formal unitarity conditions of the Hudson-Parthasarathy type: $L_{0}^{*}+L_{0}=L_{2}^{*} L_{2}, L_{2}=-L_{1}^{*} W, L_{3}=W-I, W^{*} W=W W^{*}=I$ (see [10]).

In the sequel, we assume that $(I-i K)^{-1}: \mathcal{H} \rightarrow \operatorname{dom} R^{*}$. Since $R^{*}$ is a closed operator, by the closed graph theorem ([11, Chap. $3 \S 4]), L_{1}=2 i R^{*}(2-i K)^{-1}$ is a bounded operator, and so is $L^{*} L$. Hence, the densely defined operator $L_{2}=2 i(2-i K)^{-1} R=$ $-W L_{1}^{*}$ can be extended from $\operatorname{dom} R$ to entire $\mathcal{H}$ as a bounded operator. Thus, under the assumption $(I-i K)^{-1}: \mathcal{H} \rightarrow \operatorname{dom} R^{*}$ all operators $L_{k}, 1 \leq k \leq 3$ are bounded.

The connection between the operator and its normal form ([5]) implies the explicit representation of the unitary group generated by the normal exponent (2.7):

$$
\begin{aligned}
& U_{t} h \otimes \psi(v)=\int e^{-G_{\lambda} t} d E_{\lambda} h \otimes \psi\left(e^{i \omega t}\left(e^{i Z_{\lambda} \widehat{\pi}_{[0, t)}} v+i \rho_{\lambda} e^{i \theta_{\lambda}} \frac{2}{2-i \lambda} \widetilde{I}_{[0, t)}\right)\right) \\
& \times \exp \left\{i \rho_{\lambda} e^{-i \theta_{\lambda}} \frac{2}{2-i \lambda}\left(\widetilde{I}_{[0, t)}, v\right)\right\},
\end{aligned}
$$

where $G_{\lambda}$ is the spectral density of the dissipative operator $G=-i H+R^{*}(2-i K)^{-1} R$, and $\exp \left\{i Z_{\lambda}\right\}=(2+i \lambda) /(2-i \lambda)$. By "Lemma on Four Limits", the same equations 
(2.8)-(2.9) also follows from representation (2.3) of the unitary group $U_{t}^{(\alpha)}$ as $\alpha \rightarrow 0$. The commutation rules

$$
\left[\widehat{\pi}_{A}, \widehat{\pi}_{B}\right]=0 \quad \text { for } A \cap B=\emptyset, \quad \text { and } \widehat{\pi}_{T} e^{i \omega t}=e^{i \omega t} \widehat{\pi}_{T+t}
$$

imply the group property of $U_{t}$.

The operators $A(1), A^{\dagger}(1)$ and $A^{\dagger}(1) A(1)$ can be characterized by quadratic forms acting on exponential vectors with arguments from $\widetilde{W}_{2}^{1}(\mathbb{R})$ as follows:

$$
(\psi(f), A(1) \psi(g))=\widetilde{g}(0) e^{(f, g)}, \quad\left(\psi(f), A^{\dagger}(1) A(1) \psi(g)\right)=\overline{\widetilde{f}(0)} \widetilde{g}(0) e^{(f, g)} .
$$

These unclosable quadratic forms generate well defined fundamental operator-valued set functions which are unitarily equivalent to the fundamental processes of creation, annihilation and number process introduced in [10]. We use here the following representation for these processes:

$$
\begin{gathered}
A^{+}(T)=\int_{T} \frac{d t}{\sqrt{2 \pi}} \int d \omega e^{i \omega t} a^{\dagger}(\omega)=\int_{T} d t A_{t}^{\dagger}(1), \\
A(T)=\int_{T} \frac{d t}{\sqrt{2 \pi}} \int d \omega e^{-i \omega t} a(\omega)=\int_{T} d t A_{t}(1), \\
\Lambda(T)=\int_{T} \frac{d t}{2 \pi} \iint d \omega d \nu e^{i(\omega-\nu) t} a^{\dagger}(\omega) a(\nu)=\int_{T} d t A_{t}^{\dagger}(1) A_{t}(1),
\end{gathered}
$$

where $A_{t}(1)=J_{t} A(1) J_{t}^{*}, J_{t}=e^{i t E}, E=\int_{\mathbb{R}} \omega d \omega a^{\dagger}(\omega) a(\omega)$.

Consider the operator-valued set function $u(s, t)$ (the unitary cocycle) generated by the unitary group $U_{t}$ :

$$
u(s, t)=J_{s} U_{t-s} J_{t}^{*} .
$$

Using the commutation rule $J_{t}^{*} \psi(f)=\psi\left(e^{-i \omega t} f\right)$, we immediately obtain from $(2.9)$ an explicit equation representing the action of $u(s, t)$ on tensor products $h \otimes \psi(v)$

$$
\begin{aligned}
u(T) h \otimes \psi(v)= & \int e^{-G_{\lambda} \operatorname{mes} T} d E_{\lambda} h \otimes \psi\left(e^{i Z_{\lambda} \widehat{\pi}_{T}} v+i \rho_{\lambda} e^{i \theta_{\lambda}} \frac{2}{2-i \lambda} \widetilde{I}_{T}\right) \\
& \times \exp \left\{i \rho_{\lambda} e^{-i \theta_{\lambda}} \frac{2}{2-i \lambda}\left(\widetilde{I}_{T}, v\right)\right\},
\end{aligned}
$$

where $\widetilde{I}_{T}(\omega)$ is the Fourier transform of the indicator function $I_{T}(t)$. Straightforward computation of the time derivative implies the adapted QSDE for $u(s, t)$ :

$$
\begin{gathered}
d u(s, t)=u(s, t) M\left(d t_{+}\right), \quad s-\lim _{t \rightarrow s} u(s, t)=I, \\
M(T)=L_{0} \otimes \operatorname{mes} T+L_{1} \otimes A(T)+L_{2} \otimes A^{\dagger}(T)+L_{3} \otimes \Lambda(T) .
\end{gathered}
$$

The set-function $u(T)$ has a cocycle property: $u\left(T_{1}\right) u\left(T_{2}\right)=u\left(T_{1} \cup T_{2}\right)$ for disjoint sets $T_{1}, T_{2} \subseteq \mathbb{R}$. Therefore, QSDE (2.12) can be considered as a unitary representation of the Schrödinger equation with the formal Hamiltonian (2.8):

$$
u(s, t)=J_{s} U_{t-s} J_{t}^{*}, \quad M(T)=i \int_{T} d t J_{t}\left(H_{s r s}-I \otimes E\right) J_{t}^{*} .
$$

Thus we obtain 
Theorem 1. Suppose that $K$ and $R$ are commuting operators, $K$ is a self-adjoint operator and $R$ is a closed operator such that $(I-i K)^{-1}: \mathcal{H} \rightarrow \operatorname{dom} R^{*}$. Then the family of Hamiltonians (2.1) generates the family of unitary groups $U_{t}^{(\alpha)}=e^{i t H^{(\alpha)}}$ such that

- there exists a strong limit of the family of unitary groups $U_{t}=s-\lim U_{t}^{(\alpha)}$ which is a unitary group with the formal generator $H_{\text {srs }}$ (2.8);

- there exists an adapted unitary cocycle (2.11)

$$
u(\tau, t)=s-\lim _{\alpha \rightarrow 0} J_{\tau} e^{i(t-\tau) H_{2}^{(\alpha)}} J_{t}^{*},
$$

such that $u(\tau, t)$ is a unitary solution of QSDE with the stochastic differential (2.12).

3. Symmetric boundary value problem associated to QSDE. It should be pointed out that $H_{w}$ and $H_{\text {srs }}$ are not well defined operators on the product $\mathcal{H} \otimes$ $\Gamma^{S}\left(\mathcal{L}_{2}(\mathcal{R})\right)$. They vanish in the dense set

$$
\mathcal{E}_{0}=\operatorname{span}\left\{\psi(h): h \in \widetilde{W_{2}^{1}}(\mathbb{R}), \widetilde{h}(0)=0\right\}
$$

and in this sense they are singular. Typically, there exists an implicit correspondence between singular perturbed operators and boundary value problems for these operators $([2,3,12])$. Since we have the explicit representation of the unitary group $U_{t}$, we can describe the generator rigorously. First of all let us describe the domain of the infinitesimal operator. Main observations of this Section are generalizations of the algebraic properties of the following toy boundary value problem.

For any $\lambda \in \mathbb{R}, L \in \mathbb{C}$, consider the set $\mathcal{D}_{\lambda, L}$ of vectors $F=\left\{f_{0}, f_{1}, \ldots\right\}$ with symmetric continuous components:

$$
f_{0} \in \mathscr{C}, \quad f_{n}:(\mathbb{R} \backslash\{0\})^{n} \rightarrow \mathscr{C}, \quad n=1,2, \ldots
$$

such that

where $A_{ \pm} f_{0}=0$ and

$$
\left\{\left(A_{+}-A_{-} e^{i \lambda}\right) f_{n+1}\right\}(x)=L f_{n}(x), \quad x \in(\mathbb{R} \backslash\{0\})^{n},
$$

$$
\left(A_{ \pm} f_{n+1}\right)\left(x_{1}, \ldots, x_{n}\right)=\lim _{\varepsilon \rightarrow \pm 0} f_{n+1}\left(x_{1}, \ldots, x_{n}, \varepsilon\right), \quad n=1,2, \ldots
$$

by the definition. Then, clearly, for $F, G \in \mathcal{D}_{\lambda, L}$ the following identity holds true:

$$
\begin{gathered}
\left(\left(A_{-}-A_{+}\right) \bar{g}_{n+1} f_{n+1}\right)(x)=\left.\left(\bar{g}_{n+1} f_{n+1}\right)(x)\right|_{x_{n+1}=+0} ^{x_{n+1}=-0} \\
=-|L|^{2} \bar{g}_{n}(x) f_{n}(x)-e^{-i \lambda} L\left(A_{-} \bar{g}_{n+1}\right)(x) f_{n}(x)-\bar{g}_{n}(x) \bar{L} e^{i \lambda}\left(A_{-} f_{n+1}\right)(x) .
\end{gathered}
$$

Set $\partial_{x}=\sum_{k} \frac{\partial}{\partial x_{k}}$. Integrating by parts over $(\mathbb{R} \backslash\{0\})^{n}$ the sesquilinear inner product in $L_{2}\left((\mathbb{R} \backslash\{0\})^{n}\right)$, we obtain

$$
\begin{gathered}
\left(g_{n+1}, i \partial_{x} f_{n+1}\right)=\left(i \partial_{x} g_{n+1}, f_{n+1}\right) \\
\left.-(n+1)\left\{i|L|^{2}\left(g_{n}, f_{n}\right)\right)+\left(g_{n}, i \bar{L} e^{i \lambda}\left(A_{-} f_{n+1}\right)\right)+\left(i \bar{L} e^{i \lambda}\left(A_{-} g_{n+1}\right), f_{n}\right)\right\} .
\end{gathered}
$$

Therefore, the operator

$$
\widehat{H}=H_{0}+i \partial_{x}+i|L|^{2} / 2+i \bar{L} e^{i \lambda} A_{-}, \quad H_{0} \in \mathbb{R}
$$


is symmetric on the vector subspace $\mathcal{D}_{\lambda, L}$ in the Hilbert space $\mathcal{H}$ with sesquilinear inner product

Indeed,

$$
(G, F)=\sum_{k=0}^{\infty} \frac{1}{n !}\left(g_{n}, f_{n}\right)_{L_{2}\left((\mathbb{R} \backslash\{0\})^{n}\right)} .
$$

$$
\begin{gathered}
(G, \widehat{H} F))=(G, H)\left(H_{0}+i|L|^{2} / 2\right)+\left(i \partial_{x} G, F\right)+\left(G, i \bar{L} e^{i \lambda} A_{-} F\right) \\
-i|L|^{2}(G, F)-\left(G, i \bar{L} e^{i \lambda} A_{-} F\right)+i\left(i \bar{L} e^{i \lambda} A_{-} G, F\right)=(\widehat{H} G, F) .
\end{gathered}
$$

Thus, the operator $\widehat{H}=H_{0}+i \partial_{x}+i|L|^{2} / 2+i \bar{L} e^{i \lambda} A_{-}$being restricted to the set $\left\{\left(A_{+}-\right.\right.$ $\left.\left.A_{-} e^{i \lambda}\right)-L\right\} F=0$ is a symmetric operator. Let us prove that the range of the resolvent generated by the unitary group (2.9) has a similar characterization.

Consider the vectors $\Phi$ from the range of the resolvent of unitary group $U_{t}: \Phi \in \mathbf{h}=$ $\mathcal{H} \otimes \Gamma^{S}\left(L_{2}(\mathbb{R})\right):$

$$
\begin{aligned}
\Phi=R_{\mu} h \otimes \psi(v) & =\int_{0}^{\infty} d t e^{-\mu t} U_{t} h \otimes \psi(v)=\left\{\Phi_{n}(\omega)\right\}, \\
\Phi_{n}(\cdot) \mathbb{R}^{n} & \rightarrow \mathcal{H}, \quad \omega=\left\{\omega_{1}, \ldots, \omega_{n}\right\},
\end{aligned}
$$

with the components

$$
\begin{gathered}
\Phi_{n}(\omega)=\int_{0}^{\infty} d t \exp \left\{-(\mu+G) t-L^{*} W \int_{0}^{t} \widetilde{v}(-\tau) d \tau\right\} \phi_{n, t}(\omega), \\
\phi_{n, t}(\omega)=\prod_{1}^{n}\left((W-1) \pi_{[0, t)} e^{i \omega_{k} t} v\left(\omega_{k}\right)+e^{i \omega_{k} t} v\left(\omega_{k}\right)+L \widetilde{I}_{[0, t)}\left(\omega_{k}\right)\right) h,
\end{gathered}
$$

where $L, W$ and $G$ are commuting operators described by the spectral densities

$$
L(\lambda)=2 i \rho(\lambda) e^{-i \Phi(\lambda)}(2-i \lambda)^{-1}, \quad W(\lambda)=e^{i Z(\lambda)}, \quad G(\lambda)=-i \nu(\lambda)+\rho(\lambda)^{2}(2-i \lambda)^{-1} .
$$

Let us denote by $\widetilde{\phi}_{n, t}(\tau)$ the Fourier-transformation of the function $\phi_{n, t}(\omega)$

$$
\widetilde{\phi}_{n, t}(\tau)=\prod_{1}^{n}\left((W-I) I_{[0, t)}\left(\tau_{k}\right) \widetilde{v}\left(\tau_{k}-t\right)+\widetilde{v}\left(\tau_{k}-t\right)+L I_{[0, t)}\left(\tau_{k}\right)\right) h,
$$

where $\tau=\left\{\tau_{1}, \ldots, \tau_{n}\right\}$. Let $\mathcal{K}$ be a subset in $\{1, \ldots, n\}$ and let $\mathcal{K}^{a}$ be its complement. Set

$$
P_{\mathcal{K}, t}^{(n)}(\tau)=\prod_{k \in \mathcal{K}}\left((W-I) \widetilde{v}\left(\tau_{k}-t\right)+L\right) I_{[0, t)}\left(\tau_{k}\right) \in \mathcal{B}(\mathbf{h}),
$$

then

$$
\widetilde{\phi}_{n, t}(\tau)=\sum_{\mathcal{K}}\left(P_{\mathcal{K}, t}^{(n)}(\tau) \prod_{m \in \mathcal{K}^{a}} \widetilde{v}\left(\tau_{m}-t\right)\right) h .
$$

Note that on the set $\tau \in(\mathbb{R} \backslash\{0\})^{n}$ the function $\widetilde{\phi}_{n, t}(\tau)$ depends on arguments $\tau_{k}-t$.

Clearly, the functions $P_{\mathcal{K}, t}^{(n)}(\tau)$ have discontinuities of the first kind in all hyperplanes where variables $\tau_{k}$ changes sign:

$$
\lim _{\tau_{k} \rightarrow-0} P_{\mathcal{K}, t}^{(n)}(\tau)=I_{\mathcal{K}^{a}}(k) P_{\mathcal{K}, t}^{(n)}(\tau),
$$




$$
\lim _{\tau_{k} \rightarrow+0} P_{\mathcal{K}, t}^{(n)}(\tau)=I_{\mathcal{K}^{a}}(k) P_{\mathcal{K}, t}^{(n)}(\tau)+((W-I) \widetilde{v}(-t)+L) P_{\mathcal{K} \backslash\{k\}, t}^{(n-1)}(\tau) .
$$

Therefore, Eq. (3.2) implies the following difference of substitutions:

$$
\left.P_{\mathcal{K}, t}^{(n)}(\tau)\right|_{\tau_{k}=+0} ^{\tau_{k}=-0}=-((W-I) \widetilde{v}(-t)+L) P_{\mathcal{K} \backslash\{k\}, t}^{(n-1)}(\tau) I_{\mathcal{K}}(k) .
$$

Let us calculate the jump of $\widetilde{\phi}_{n, t}(\tau)$ at the points where $\tau_{k}$ changes sign. Note that

$$
\lim _{\tau_{k} \rightarrow-0} \widetilde{\phi}_{n, t}(\tau)=\widetilde{v}(-t) \widetilde{\phi}_{n-1, t}\left(\tau_{1}, \ldots, \tau_{k-1}, \tau_{k+1}, \ldots, \tau_{n}\right) .
$$

Taking into account Eqs. (3.1) and (3.3), we find the jumps in amplitude and phase of the functions from the domain of the infinitesimal operator of the group $U_{t}$ :

$$
\lim _{\tau_{k} \rightarrow+0} \widetilde{\phi}_{n, t}(\tau)=W \lim _{\tau_{k} \rightarrow-0} \widetilde{\phi}_{n, t}(\tau)+L \widetilde{\phi}_{n-1, t}\left(\tau_{1}, \ldots, \tau_{k-1}, \tau_{k+1}, \ldots, \tau_{n}\right) .
$$

Let $\mathcal{D}_{W, L}=D \otimes \Gamma^{S}\left(\widetilde{W}_{2}^{1}(\mathbb{R} \backslash\{0\})\right)$ be a vector subspace in $\mathbf{h}$ with the elements satisfying assumption (3.4), and let $A\left(\delta_{ \pm}\right), \Lambda\left(\delta_{ \pm}\right), \widehat{N}$ be operators acting on Fock vectors by the rules

$$
\begin{gathered}
\left(\Phi, \Lambda\left(\delta_{ \pm}\right) \Psi\right)=\left.\lim _{\varepsilon \rightarrow+0} \sum_{1}^{\infty} \frac{1}{n !} \sum_{k=1}^{n} \int_{(\mathbb{R} \backslash\{0\})^{n-1}} \prod_{m \neq k} d \tau_{m}\left(\widetilde{\Phi}_{n}, \widetilde{\Psi}_{n}\right)_{\mathcal{H}}\right|_{\tau_{k}= \pm \varepsilon}, \\
\mathcal{F}_{\omega \rightarrow \tau}\left(A\left(\delta_{ \pm}\right) \Psi\right)_{n}(\tau)=\left.\lim _{\varepsilon \rightarrow+0} \sum_{k=1}^{n} \widetilde{\Psi}_{n+1}(\tau)\right|_{\tau_{k}= \pm \varepsilon}, \quad \widehat{N} \Psi_{n}(\omega)=n \Psi_{n}(\omega) .
\end{gathered}
$$

In this notation, the boundary condition (3.4) in the symmetric Fock space $\Gamma^{S}$ looks as follows

$$
(\widehat{N}+1)^{-1}\left(I \otimes A\left(\delta_{+}\right)-W \otimes A\left(\delta_{-}\right)\right) \Psi=L \otimes I \Psi .
$$

This condition is fulfilled for components $\Psi_{1}, \Psi_{2}, \ldots$ of the Fock vectors $\Phi \in \mathcal{D}_{W, L}$.

Let us prove that the operator

$$
\widehat{H}=i G \otimes I+I \otimes \widehat{E}+i L^{*} W \otimes A\left(\delta_{-}\right), \quad \widehat{E}=\int_{\mathbb{R} \backslash\{0\}} d \tau a^{+}(\tau) a(\tau) i \partial_{\tau},
$$

is symmetric in $\mathcal{D}_{W, L}$. Let $\Phi, \Psi \in \mathcal{D}_{W, L}$ and let $B$ be a Hermitian operator such that $\operatorname{dom} B \otimes I \supseteq \mathcal{D}_{W, L}$. The integration by parts gives an identity, where the difference of substitutions is expressed through the operators $\Lambda\left(\delta_{ \pm}\right)$:

$$
\begin{aligned}
& (\Phi, B \otimes \widehat{E} \Psi)-(B \otimes \widehat{E} \Phi, \Psi)=i\left(\Phi, B \otimes\left(\Lambda\left(\delta_{-}\right)-\Lambda\left(\delta_{+}\right)\right) \Psi\right) \\
& \quad=\left.i \sum_{1}^{\infty} \frac{1}{n !} \sum_{k=1}^{n} \int_{(\mathbb{R} \backslash\{0\})^{n-1}} \prod_{m \neq k} d \tau_{m}\left(\widetilde{\Phi}_{n}(\tau), B \widetilde{\Psi}_{n}(\tau)\right)_{\mathcal{H}}\right|_{\tau_{k}=+0} ^{\tau_{k}=-0}
\end{aligned}
$$

By using the boundary condition (3.5) for functions $\widetilde{\phi}_{n}$ and $\widetilde{\psi}_{n}$, we find the difference of substitutions of the integral (3.7):

$$
\begin{aligned}
& I\left(\Phi, B \otimes\left(\Lambda\left(\delta_{+}\right)-\Lambda\left(\delta_{-}\right)\right) \Psi\right)=i\left(\Phi,\left(W^{*} B W-B\right) \Lambda\left(\delta_{)}-\Psi\right)\right. \\
& \quad+i(L \Phi, B L \Psi)+I\left(W A\left(\delta_{-}\right) \Phi, B L \Psi\right)+i\left(L \Phi, B W A\left(\delta_{-}\right) \Psi\right) .
\end{aligned}
$$

In particular, Eq. (3.8) is simplified for $B=I$ :

$I\left(\Phi, I \otimes\left(\Lambda\left(\delta_{+}\right)-\Lambda\left(\delta_{-}\right)\right) \Psi\right)=i\left(\Phi, L^{*} L \Psi\right)-\left(i L^{*} W \otimes A\left(\delta_{-}\right) \Phi, \Psi\right)+\left(\Phi, i L^{*} W \otimes A\left(\delta_{-}\right) \Psi\right)$. 
Taking into account the identity $i G-i L^{*} L=(i G)^{*}$, we obtain from here an identity which means the symmetry property of the operator $\widehat{H}$ in $\mathcal{D}_{W, L}$ :

$$
\begin{gathered}
(\Phi, \widehat{H} \Psi)=\left((I \otimes \widehat{E} \Phi, \Psi)+\left(\Phi,\left\{i G \otimes I+i L^{*} W \otimes A\left(\delta_{-}\right)\right\} \Psi\right)\right. \\
-i\left(\Phi, I \otimes\left(\Lambda\left(\delta_{+}\right)-\Lambda\left(\delta_{-}\right)\right) \Psi\right) \\
=\left((I \otimes \widehat{E} \Phi, \Psi)+\left(\Phi,(i G)^{*} \otimes I \Psi\right)+\left(i L^{*} W \otimes A\left(\delta_{-}\right) \Phi, \Psi\right)=(\widehat{H} \Phi, \Psi) .\right.
\end{gathered}
$$

Let us find how the generator of the group $U_{t}$ acts on vectors belonging to the resolvent. Set $\Psi \in \mathbf{h}, \Phi=R_{\mu} h \otimes \psi(v)$. By the definitions of the generator, we have

$$
\begin{aligned}
& (\Psi, \widehat{H} \Phi)=\lim _{s \rightarrow+0} \frac{1}{i} \frac{d}{d s}\left(\Psi, U_{s} \Phi\right)=\frac{1}{i} \int_{0}^{\infty} d t \sum_{n=0}^{\infty} \frac{1}{n !} \int_{(\mathbb{R} \backslash\{0\})^{n}} d \tau \\
& \times\left.\left(\widetilde{\psi}_{n}(\tau), \frac{d}{d s} e^{-(G+\mu) t-G s-i L^{*} W}{ }_{0}^{\mathrm{T}_{+s}} \widetilde{v}(-\tau) d \tau \widetilde{\phi}_{n, t+s}(\tau)\right)_{\mathcal{H}}\right|_{s=0} .
\end{aligned}
$$

As it was observed earlier, the functions $\widetilde{\phi}_{n, t}(\tau)$ depend on differences $\tau_{k}-t$. Therefore,

$$
\frac{d}{d t} \widetilde{\phi}_{n, t}(\tau)=-\sum_{k=1}^{n} \frac{\partial}{\partial \tau_{k}} \widetilde{\phi}_{n, t}(\tau)=i \widehat{E}_{\phi_{n, t}}(\tau) .
$$

On the other hand,

$$
A\left(\widetilde{\left.\delta_{-}\right)} \phi_{n, t}(\tau)=n \widetilde{v}(-t) \phi_{n-1, t}(\tau)\right.
$$

by definition of the operator $A\left(\delta_{-}\right)$. Now, taking into account definition (3.9) and identities (3.10) and (3.11), we obtain

$$
\begin{aligned}
& (\Psi, \widehat{H} \Phi)_{\mathbf{h}}=\int_{0}^{\infty} d t\left(\psi_{0}, i G e^{-(G+\mu) t-i L^{*} W}{ }_{0}^{\mathrm{T}} \widetilde{v}(-\tau) d \tau h\right)_{\mathcal{H}}+\int_{0}^{\infty} d t \sum_{n=1}^{\infty} \frac{1}{n !} \int_{(\mathbb{R} \backslash\{0\})^{n}} d \tau \\
& \times\left(\widetilde{\psi}_{n}(\tau), e^{-(G+\mu) t-i L^{*} W}{ }_{0}^{\mathrm{T}} \widetilde{v}(-\tau) d \tau\left(i G+i L^{*} W \widetilde{v}(-t)+I \sum_{k=1}^{n} \frac{\partial}{\partial \tau_{k}}\right) \widetilde{\phi}_{n, t}(\tau)\right)_{\mathcal{H}} \\
& =\left(\Psi,\left\{i G+i L^{*} W \otimes A\left(\delta_{-}\right)+I \otimes \widehat{E}\right\} \Phi\right)_{\mathbf{h}},
\end{aligned}
$$

that is the generator $\widehat{H}$ of the group $U_{t}$ has the form (3.6). Thus, we have proved the following theorem.

THEOREM 2. The operator

$$
\widehat{H}=i G \otimes I+I \otimes \widehat{E}+i L^{*} W \otimes A\left(\delta_{-}\right),
$$

where $G=i H+\frac{1}{2} L^{*} L, H=\frac{1}{4} L^{*} K L-H_{0}$, is symmetric in $\mathcal{D}_{W, L}$. It generates the one-parameter unitary group $U_{t}(2.9)$.

It is important to stress that the proof of the symmetry property does not assume that the operators $L, G$ and $W$ commute. The proof can be easily extended to operators of the form

$$
\widehat{H}=i G+I \otimes \widehat{E}+i \sum_{l, m} L_{l}^{*} W_{l, m} \otimes A_{m}\left(\delta_{-}\right)
$$


with the boundary condition

$$
(\widehat{N}+1)^{-1}\left(I \otimes A_{l}\left(\delta_{+}\right)-\sum_{m} W_{l, m} \otimes A_{m}\left(\delta_{-}\right)\right) \Psi=L_{l} \otimes I \Psi
$$

where $W=\left\{W_{l, m}\right\}$ is an $(M \times M)$-matrix with coefficients in $\mathcal{B}(\mathcal{H})$ such that $W^{*} W=I$, and $\left\{A_{m}(g): g \in L_{2}(\mathbb{R}), 1 \leq m \leq M\right\}$ are the annihilation operators in $\Gamma^{S}\left(L_{2}\left(\mathbb{R}^{M}\right)\right)$ which commute for different $l$.

4. Creation and annihilation processes of the second order. In the general case, it is a difficult problem to find the matrices $\mathcal{M}_{t}$ and $\mathcal{N}_{t}$ defined by (1.3). Nevertheless, equations (1.3) can be used to obtain the asymptotics of the solution as $t \rightarrow 0$ and to find the normal form of the strong resolvent limit of the family of Hamiltonians (1.1). This requires the asymptotical expansions of $\mathcal{M}_{t}$ and $\mathcal{N}_{t}$. In the sequel we consider a degenerating sequence of quadratic selfadjoint Hamiltonians

$$
\begin{aligned}
H_{2}^{(\alpha)} & =\frac{Q}{2} A^{\dagger}\left(h_{\alpha}\right) A^{\dagger}(h)+\frac{Q^{*}}{2} A\left(h_{\alpha}\right) A(h)+I \otimes E+H_{0} \otimes I \\
& +R \otimes A^{\dagger}\left(f_{\alpha}\right)+R^{*} \otimes A\left(f_{\alpha}\right)+K \otimes A^{\dagger}\left(g_{\alpha}\right) A\left(g_{\alpha}\right),
\end{aligned}
$$

with $f, g, h \in L_{2, \widetilde{1}}^{+}(\mathbb{R}), L_{2, \widetilde{1}}^{+}(\mathbb{R})=\left\{g: \widetilde{g} \geq 0, \widetilde{g} \in L_{2} \cap L_{1}\right\}$, where $Q \in \mathcal{C}(\mathcal{H}), h_{\alpha}(\omega)=$ $h(\omega \alpha), f(0)=g(0)=h(0)=(2 \pi)^{-1 / 2}$. Thus, the weak limit $H_{w}$ equals

$$
H_{w}=I \otimes E+H_{0} \otimes I+K \otimes A^{\dagger}(1) A(1)+\mathcal{R} A^{\dagger}(1)+\mathcal{R}^{*} A(1)
$$

where $\mathcal{R}=R \otimes I+Q \otimes A^{\dagger}(h) / 2$ and

$$
\mathcal{R} A^{\dagger}(1)=w-\lim \left(R \otimes A^{\dagger}\left(f_{\alpha}\right)+Q \otimes A^{\dagger}(h) A^{\dagger}\left(h_{\alpha}\right) / 2\right)=\left(R \otimes I+Q / 2 \otimes A^{\dagger}(h)\right) A^{\dagger}(I) .
$$

The natural conjecture is that the strong resolvent limit $H_{s r s}\left(a^{*}, a\right)$ corresponding to Hamiltonian (4.1) can be obtained from (2.8) by the change $R \rightarrow \mathcal{R}$.

To evaluate the normal form $H_{\text {srs }}\left(a^{*}, a\right)$ of the strong resolvent limit, it suffices to study the small $t$ asymptotic expansion of the functions $\mu_{t}^{(\alpha)}$ and $\lambda_{t}^{(\alpha)}$ described by the system of ODE

$$
\begin{cases}\dot{\bar{\mu}}_{t}^{(\alpha)}=i H_{a a}^{(\alpha)}(t) \lambda_{t}^{(\alpha)}, & \bar{\mu}^{(\alpha)}{ }_{0}=0, \\ \dot{\lambda}_{t}^{(\alpha)}=-i \bar{H}_{a a}^{(\alpha)}(t) \bar{\mu}_{t}^{(\alpha)}, & \lambda_{0}^{(\alpha)}=I,\end{cases}
$$

where $H_{a a}^{(\alpha)}(t)=P_{-t}^{(\alpha)} H_{a a}^{(\alpha)} P_{-t}^{(\alpha)}$. The operators $\lambda_{t}$ and $\mu_{t}$ are related to $\Phi_{t}$ and $\Psi_{t}$ by the interaction representation generated by $P_{t}^{(\alpha)}$ :

$$
\lambda_{t}=P_{t}^{(\alpha)} \Phi_{t}, \quad \mu_{t}=P_{t}^{(\alpha)} \Psi_{t}, \quad \Phi_{t}^{-1} \Psi_{t}=\lambda_{t}^{-1} \mu_{t}, \quad \bar{\Psi}_{t} \Phi_{t}^{-1}=P_{t} \bar{\mu}_{t} \lambda_{t}^{-1} P_{t} .
$$

The solutions of this system, being rewritten as left cocycles, read as follows

$$
\begin{gathered}
\bar{\mu}_{t}^{(\alpha)}=-i\left(\int_{0}^{t} d s_{1} H_{a a}^{(\alpha)}\left(s_{1}\right)+\int_{0}^{t} d s_{1} \int_{0}^{s_{1}} d s_{2} \int_{0}^{s_{2}} d s_{3} H_{a a}^{(\alpha)}\left(s_{1}\right) \overline{H_{a a}^{(\alpha)}}\left(s_{2}\right) H_{a a}^{(\alpha)}\left(s_{3}\right)+\ldots\right), \\
\lambda_{t}^{(\alpha)}=I+\int_{0}^{t} \int_{0}^{s_{1}} d s_{1} d s_{2} \overline{H_{a a}^{(\alpha)}}\left(s_{1}\right) H_{a a}^{(\alpha)}\left(s_{2}\right)+\ldots
\end{gathered}
$$


By using "Lemma on Four Limits" and expansions (4.2), we prove that if $(I-i K)^{-1}$ : $\mathcal{H} \rightarrow \operatorname{dom} Q^{*} \cap \operatorname{dom} R^{*}$, then the following asymptotic expansions hold true as $\alpha \rightarrow 0$ :

$$
\begin{gathered}
\lim \lambda_{t}^{(\alpha)}=I+\gamma_{t}, \quad \gamma_{t}=\int_{0}^{t} d s\left|h_{s}\right\rangle Q^{*}(2-i K)^{-1} Q\left\langle h_{s}\right|+O\left(t^{2}\right), \\
\lim \mu_{t}^{(\alpha)}=-i \int_{0}^{t} d s\left\{\left|h_{s}\right\rangle(2-i K)^{-1} Q\left\langle\widetilde{I}_{[0, s)}|+| \widetilde{I}_{[0, s)}\right\rangle(2-i K)^{-1} Q\left\langle h_{s}\right|\right\}+O\left(t^{2}\right),
\end{gathered}
$$

where $h_{t}=e^{i \omega t}\left(I+(W-I) \widehat{\pi}_{(0, t)}\right) h(\omega),\left\|h_{t}\right\|=\|h\|$. Hence, $\Phi_{t}^{-1} \Psi_{t}=\bar{\mu}_{t}+O\left(t^{2}\right)$ and

$$
\begin{gathered}
\lim \operatorname{det}\left(\Phi_{t}^{(\alpha)} P_{t}^{(\alpha)}\right)=\lim \exp \left\{\operatorname{Tr} \ln \lambda_{t}^{(\alpha)}\right\}=1+\operatorname{Tr}\left\{\gamma_{t}\right\}+O\left(t^{2}\right) \\
=1+t\|h\|^{2} Q^{*}(2-i K)^{-1} Q+O\left(t^{2}\right), \\
\lim i \int_{0}^{t} d \tau H_{a}^{(\alpha)}\left(\Phi_{\tau}^{(\alpha)}\right)^{-1} g_{\tau}^{(\alpha)}=t R^{*}(2-i K)^{-1} R+O\left(t^{2}\right), \\
\lim i \int_{0}^{t} d \tau g_{\tau}^{(\alpha)}\left(\Phi_{\tau}^{(\alpha)}\right)^{-1} H_{a a}^{(\alpha)}\left(\Phi_{\tau}^{(\alpha)}\right)^{-1} g_{\tau}^{(\alpha)}=O\left(t^{2}\right), \\
+\left(a^{*}, h\right)\left(a^{*}, e^{i \omega t} \widetilde{I}_{[0, t)}\right)(I-i K / 2)^{-1} Q+(\bar{h}, a)\left(e^{i \omega t} \widetilde{I}_{[0, t)} a\right)(I-i K / 2)^{-1} Q^{*}, \\
\lim \left(\begin{array}{c}
a \\
a^{*}
\end{array}\right)^{\prime} \mathcal{N}_{t}\left(\begin{array}{c}
a \\
\bar{g}_{t}-\bar{\Psi}_{t} \Phi_{t}^{-1} g_{t} \\
-\Phi_{t}^{-1} g_{t}
\end{array}\right)=\left(\begin{array}{c}
a \\
a^{*}
\end{array}\right)^{\prime} \mathcal{N}_{t}\left(\begin{array}{c}
a \\
\bar{g}_{t} \\
-\Phi_{t}
\end{array}\right)=\left(W g_{t}\right)+O\left(t^{2}\right)= \\
i(I-i K / 2)^{-1}\left\{R^{*}\left(\widetilde{I}_{[0, t)}+Q / 2 \bar{h}, a\right)+R\left(e^{i \omega t} \widetilde{I}_{[0, t)}+Q^{*} / 2 \bar{h}, a\right)\right\}+O\left(t^{2}\right) .
\end{gathered}
$$

These expansions gives an equation for $H_{\text {srs }}$ similar to the previous one and confirm the above conjecture:

$$
\begin{gathered}
i H_{\text {srs }}=\mathcal{L}_{0}+(W-I) A^{\dagger}(1) A(1)+\mathcal{L} A^{\dagger}(1)-\mathcal{L}^{*} W A(1), \\
\mathcal{L}_{0}=i\left(H_{0} \otimes I+I \otimes E\right)-\mathcal{R}^{*}(2-i K)^{-1} \mathcal{R}, \quad \mathcal{L}=i(1-i K / 2)^{-1} \mathcal{R}, \\
\mathcal{R}=R \otimes I+Q \otimes A^{\dagger}(h) / 2
\end{gathered}
$$

with the same $K$ and $W$ as above. We stress that the limits $H_{w}$ and $H_{\text {srs }}$ are different.

To pass to a quantum stochastic differential equation, we cancel out the term $I \otimes E$ from the Hamiltonian by passing to the interaction representation, and evaluate the stochastic differential:

$$
M(T)=i \int_{T} d t J_{t}\left(H_{s r s}-I \otimes E\right) J_{t}^{*}
$$

Thus, the contribution to the singular components of $H_{s r s}$ is given by the stochastic processes of creation and annihilation of the second order:

$$
A_{2}^{\dagger}(T)=\int_{T} d t A^{\dagger}\left(h e^{i \omega t}\right) A^{\dagger}\left(d t_{+}\right), \quad A_{2}(T)=\int_{T} d t A\left(h e^{-i \omega t}\right) A\left(d t_{+}\right),
$$

where $A\left(h e^{-i \omega t}\right)$ acts as a time-dependent annihilation operator; it multiplies the coherent 
vectors by the convolution of the Fourier transformations of $h$ and $g$ :

$$
A\left(h e^{-i \omega t}\right) \psi(g)=\int d \tau \widetilde{\widetilde{h}}(\tau) \widetilde{g}(t+\tau) \psi(g) .
$$

In the particular case when $\widetilde{h}$ is supported by $\mathbb{R}_{-}$, the processes $A_{2}(T)$ and $A_{2}^{\dagger}(T)$ are adapted. Fortunately, in the nonadapted case, there is no problem to define rigorously the stochastic integrals (4.6) because they contain products of commuting operators.

The associative Ito multiplication table for the operator-valued set-functions $A(T)$, $\Lambda(T), A_{2}(T)$ and adjoint families follows in the standard way from CCR (see [13, 14]):

\begin{tabular}{|l||c|c|c|}
\hline & $d A^{\dagger}$ & $d \Lambda$ & $d A_{2}^{\dagger}$ \\
\hline \hline$d A$ & $d t$ & $d A$ & $A^{\dagger}\left(h_{t}\right) d t$ \\
\hline$d \Lambda$ & $d A^{\dagger}$ & $d \Lambda$ & $d A_{2}^{\dagger}$ \\
\hline$d A_{2}$ & $A\left(h_{t}\right) d t$ & $d A_{2}$ & $\nu_{t} d t$ \\
\hline
\end{tabular}

where $h_{t}=e^{-i \omega t} h$ and $\nu_{t}=A^{\dagger}\left(h_{t}\right) A\left(h_{t}\right)+\|h\|^{2}=A\left(h_{t}\right) A^{\dagger}\left(h_{t}\right)$. All the products which are not indicated in the table are zero.

Assume that $L_{k}$ are bounded operators from $\mathcal{B}(\mathcal{H}) \otimes \Gamma^{S}\left(L_{2}(\mathbb{R})\right)$ and

$$
M(T)=L_{0} \operatorname{mes} T+L_{1} A^{\dagger}(T)+L_{2} A(T)+L_{3} \Lambda(T)+L_{4} A_{2}(T)+L_{5} A_{2}^{\dagger}(T) .
$$

Then the conditions necessary for the cocycle satisfying the QSDE

$$
d u(s, t)=u(s, t) M\left(d t_{+}\right)
$$

to be unitary can be derived from the equations $M(d t)+M^{*}(d t)+M^{*}(d t) M(d t)=0$, $M(d t)+M^{*}(d t)+M(d t) M^{*}(d t)=0$. These conditions and the Ito multiplication table imply the equations for the coefficients $L_{k}$ :

$$
\begin{gathered}
L_{0}+L_{0}^{*}+L_{1}^{*} L_{1}+L_{5}^{*} L_{5} \nu_{t}+L_{1}^{*} L_{5} A^{\dagger}\left(h_{t}\right)+L_{5}^{*} L_{1} A\left(h_{t}\right)=0, \\
L_{1}+L_{2}^{*}+L_{3}^{*} L_{1}=0, \quad L_{2}+L_{1}^{*}+L_{1}^{*} L_{3}=0, \\
L_{3}+L_{3}^{*}+L_{3}^{*} L_{3}=0, \quad L_{3}+L_{3}^{*}+L_{3} L_{3}^{*}=0, \\
L_{5}+L_{4}^{*}+L_{3}^{*} L_{5}=0, \quad L_{4}+L_{5}^{*}+L_{5}^{*} L_{3}=0 .
\end{gathered}
$$

The system (4.8) is similar to the equations derived in [10] to describe the conditions necessary for the solution of QSDE to be unitary. It has an explicit solution parameterized by a Hermitian operator $H_{1}$, a unitary operator $W$, and arbitrary operators $L$ and $M$ :

$$
\begin{gathered}
L_{1}=L, \quad L_{2}=-L^{*} W, \quad L_{3}=W-I, \quad L_{4}=-M^{*} W, \quad L_{5}=M, \\
L_{0}=i H_{1}-\frac{1}{2}\left(( L + M A ^ { \dagger } ( h _ { t } ) ) ^ { * } \left(\left(L+M A^{\dagger}\left(h_{t}\right)\right) .\right.\right.
\end{gathered}
$$

These coefficients coincide with the coefficients of set-function (4.7) provided

$$
\begin{gathered}
L=i(I-i K / 2)^{-1} R, \quad M=i(I-i K / 2)^{-1} Q, \\
H_{1}=H_{0}-\left(( R + Q A ^ { \dagger } ( h _ { t } ) ) ^ { * } \frac { 2 K } { 4 + K ^ { 2 } } \left(\left(R+Q A^{\dagger}\left(h_{t}\right)\right) .\right.\right.
\end{gathered}
$$

The last correction $H_{1}-H_{0}$ to $H_{0}$ follows from the strong resolvent limit of the family of "physical" Hamiltonians (4.1) and violates the normal form of the generator, but the difference $H_{1}-H_{0}$ cannot be derived within the quantum stochastic calculus. 
The symmetric boundary value problem corresponding to the formal Hamiltonian operator (4.5) is formulated similarly to Theorem 3.1. Our main result is the following theorem.

THEOREM 3. Let the operators $K, R$ and $Q$ commute, let $K$ be a self-adjoint operator, $R$ and $Q$ be closed operators such that $(I-i K)^{-1}: \mathcal{H} \rightarrow \operatorname{dom} Q^{*} \cap \operatorname{dom} R^{*}$. Then the family of Hamiltonians (4.1) generates the family of unitary groups $U_{t}^{(\alpha)}=e^{i t H^{(\alpha)}}$ such that

- there exists the strong limit of the family of unitary groups $U_{t}=s-\lim _{\alpha \rightarrow 0} U_{t}^{(\alpha)}$; $U_{t}$ is a unitary group generated by the operator

$$
\mathbf{H}=\mathcal{L}_{0}-\mathcal{L}^{*} W A\left(\delta_{-}\right)
$$

with coefficients (4.5) which is a symmetric operator on the closed linear subspace of Fock vectors $\Psi$ such that

$$
(\widehat{N}+1)^{-1}\left(I \otimes A\left(\delta_{+}\right)-W \otimes A\left(\delta_{-}\right)\right) \Psi=\mathcal{L} \Psi
$$

- there exists a nonadapted unitary cocycle $u(\tau, t)=J_{\tau} U_{t-s} J_{t}^{*}$ such that $u(\tau, t)$ is a unitary solution of $Q S D E$

$$
d u_{t}=u_{t} M\left(d t_{+}\right), \quad s-\lim _{t \rightarrow s} u(s, t)=I
$$

with the nonadapted stochastic differential

$$
M(T)=\int_{T} \mathcal{G}_{t} \otimes I d t+(W-I) \otimes \Lambda\left(d t_{+}\right)+\mathcal{L}_{t} \otimes A^{\dagger}\left(d t_{+}\right)-\mathcal{L}_{t}^{*} W \otimes A\left(d t_{+}\right),
$$

with $\mathcal{G}_{t}=i H_{0} \otimes I-\mathcal{R}_{t}^{*}(2-i K)^{-1} \mathcal{R}_{t}$, where $\mathcal{L}_{t}=i(1-i K / 2)^{-1} \mathcal{R}_{t}$ and

$$
\mathcal{R}_{t}=R \otimes I+Q \otimes A^{\dagger}\left(h e^{i \omega t}\right) / 2 \text {. }
$$

Acknowledgements. The authors wish to express their gratitude to Professors L. Accardi, V. P. Belavkin, V. D. Koshmanenko and I. Volovich for stimulating discussions of the topics related to this paper.

\section{References}

[1] L. Accardi, Y.-G. Lu and I. Volovich, Non-linear Extensions of Classical and Quantum StochastCalculus and Essentially Infinite Dimensional Analysis, V. Volterra Center, Università di Roma Tor Vergata, Preprint No 268, 1996.

[2] S. Albeverio, F. Gesztesy and R. Hoegh-Krohn, Solvable models in Quantum mechanics, Springer-Verlag, New-York, Berlin, London, 1988.

[3] S. Albeverio, W. Karwowski and V. D. Koshmanenko, Square powers of singularly perturbed operators, Math. Nachr., Vol. 173, 1995, 5-24.

[4] V. P. Belavkin and P. Staszewski, Nondemolition observation of a free quantum particle, Phys. Rev. A, Vol. 45, 1992, 1347-1356.

[5] F. A. Berezin, The Method of Second Quantization, Academic Press, New-York, 1996.

[6] A. M. Chebotarev, Quantum stochastic differential equation as a strong resolvent limit of the Schrödinger evolution, in: IV Simposio de Probabilidad y Procesos Estochasticos, Gunajuato, Mexico, 1996, Societad Mat. Mexicana, 1996, 71-89. 
[7] A. M. Chebotarev, Symmetric form of the Hudson-Parthasarathy equation, Mathematical Notes, Vol. 60, N5, 1996, 544-561.

[8] A. M. Chebotarev, Quantum stochastic differential equation is unitarily equivalent to a boundary value problem for the Schrödinger equation, Mathematical Notes, Vol. 61, N4, 1997, 510-519.

[9] C. W. Gardiner and M. J. Collett, Input and output in damped quantum systems: quantum statistical differential equations and the master equation, Phys. Rev. A, Vl. 31, 1985, 3761-3774.

[10] R. L. Hudson and K. R. Parthasarathy, Quantum Ito's formula and stochastic evolutions, Commun. Math. Phys., Vol. 93, N3, 1984, 301-323.

[11] T. Kato, Perturbation Theory for Linear Operators, 2nd ed., Springer-Verlag, BerlinHeidelberg-New York, 1980.

[12] V. D. Koshmanenko, Singular Bilinear Forms in Perturbation Theory of Self-Adjoint Operators [in Russian], Naukova dumka, Kiev, 1993.

[13] P. A. Meyer, Quantum probability for probabilists, Lecture Notes in Math., vol. 1338, 1993.

[14] K. R. Parthasarathy, An introduction to quantum stochastic calculus, Birkhäuser, Basel, 1992.

[15] P. Zoller and C. W. Gardiner, Quantum Noise in Quantum Optics: the Stochastic Schödinger Equation, To appear in: Lecture Notes for the Les Houches Summer School LXIII on Quantum Fluctuations in July 1995, Edited by E. Giacobino and S. Reynaud, Elsevier Science Publishers B.V., 1997. 